\title{
The Efficacy of Pyogenic Cavity Aerobic Therapy with Negative Pressure Drainage in the Treatment of Deep Neck Space Infections
}

\author{
Chunhui Tian $\mathbb{D}^{1,2, *}$ \\ Renwu Zhao (iD ${ }^{2, *}$ \\ Zengyu Qiu (D) \\ Yehai Liu (iD) ${ }^{2}$ \\ 'Department of Otolaryngology-Head \\ and Neck Surgery, Suzhou Hospital of \\ Anhui Medical University, Suzhou, \\ People's Republic of China; ${ }^{2}$ Department \\ of Otolaryngology-Head and Neck \\ Surgery, First Affiliated Hospital of Anhui \\ Medical University, Hefei, People's \\ Republic of China \\ *These authors contributed equally to \\ this work
}

\begin{abstract}
Objective: To determine the effectiveness of pyogenic cavity aerobic therapy with negative pressure drainage in the treatment of deep neck space infections (DNSI).

Methods: The study was a prospective, observational analysis of 36 cases of DNSI at a tertiary care center. The patients were divided into two groups according to the treatment method. Group A was treated with pyogenic cavity aerobic therapy with negative pressure drainage and included 13 patients (6 males and 7 females), while group B was treated with traditional incision debridement drainage and included 23 patients (12 males and 11 females). The average hospitalization days and doctors' workload (ie, average days of postoperative dressing changes) were analyzed and compared between the two groups.

Results: The mean hospitalization days in the traditional dressing group were $26.74 \pm 3.39$ days, while the average days of postoperative dressing change were $25.91 \pm 3.43$ days. In contrast, the averages for hospitalization days and days of postoperative dressing changes in the pyogenic cavity aerobic therapy plus negative pressure drainage were $11.08 \pm 2.11$ and $3.69 \pm 0.21$ days, respectively. All 36 patients were cured. Compared with the group B, group A had a shorter hospital stays and lower doctor workloads $(P<0.001)$.

Conclusion: Pyogenic cavity aerobic therapy is an effective and simple method for changing dressings after DNSI. This therapy, when combined with negative pressure drainage, shortens hospitalization days and days that require a dressing change. This has reduced the workload of clinical doctors and pain experienced by patients. This therapy also has a high degree of safety and a very satisfactory curative effect.
\end{abstract}

Keywords: deep neck infections, neck abscess, therapy, drainage

\section{Introduction}

Many fascial spaces with loose tissue structure in the maxillofacial and neck regions are continuous with each other, which potentially facilitates the spread of infections. ${ }^{1}$ Deep neck space infections (DNSI) are infections of the deep neck space and fascia plane, which can induce cellulitis and even formation of an abscess. DNSI are a common emergency in otorhinolaryngology and head and neck surgical departments. If not given effective and timely treatment, a multi-space fusion infection may develop rapidly and induce complications such as dyspnea, carotid sheath infection, laryngeal edema, mediastinitis, mediastinal abscess and intracranial infection. ${ }^{2,3}$ In severe cases, these infections are life-threatening. ${ }^{4,5}$

The standard treatment of deep fascial space infections includes medical support for patients, surgical drainage of the infection and correct use of antibiotics. ${ }^{4}$ With
Correspondence: Yehai Liu

Department of Otolaryngology-Head and Neck Surgery, First Affiliated Hospital of Anhui Medical University, 218 Ji-xi road, Hefei, People's Republic of China Tel +8613856906986

Email liuyehai@ahmu.edu.cn 
early administration of antibiotics, there has been a decline in the incidence of this type of disease in recent years. Surgical incision combined with debridement and drainage has been the preferred treatment option for neck multiple space infections that are difficult to control with antibiotics. ${ }^{6}$

However, clinicians must address several persistent problems, such as long-term hospital stays and antibiotic use and burdensome dressing changes. Pyogenic cavity aerobic therapy with negative pressure drainage is a new technique for treating DNSI. To the best of our knowledge, the use of this technology for treating DNSI has not been reported although we have anecdotal evidence from clinical studies of its therapeutic effect.

\section{Materials and Methods Study Design and Setting}

This study collected hospital records of 36 subjects with DNSI who were admitted to the Department of Otolaryngology - Head and neck surgery of the First Affiliated Hospital of Anhui Medical University from November 2017 to October 2019 (Table 1). We have read the Helsinki Declaration and the study was carried out in accordance with the Declaration of Helsinki. The patient data were anonymized and maintained with confidentiality. The patients were divided into two groups based on the treatment methods they received. The patients in Group A (13 patients, 6 males and 7 females, mean age $56.77 \pm 12.74 \mathrm{yr}$ ) were treated with pyogenic cavity aerobic therapy with negative pressure drainage, while those in group B (23 patients, 12 males and 11 females, average age $55.09 \pm 14.11 \mathrm{yr}$ ) were treated with traditional incision debridement drainage. There was no significant difference in gender, age, or disease condition between the two groups $(P>0.05)$, indicating comparability. There were 21 cases $(58.3 \%)$ of type 2 diabetes mellitus, 2 cases of hypertension, 1 case with a hematological disease, and 1 case with panniculitis. Twenty-eight cases underwent a tracheotomy.
All the cases underwent emergency surgery within 24 hours after admission. The maximum diameter of the abscess on computed tomography (CT) exceeded $2.5 \mathrm{~cm}$. All patients included in the study provided informed consent and agreed to the use of their medical history information. The study was approved by the local ethics committee. Exclusion criteria included a peritonsillar abscess, a pharyngeal leakage or esophageal fistula, a post traumatic infected wound, or an infection secondary to a malignant tumor.

\section{Surgical Procedures}

All patients underwent laboratory tests, an electrocardiogram, and CT imaging of the neck (Figure 1A and B). According to the general physical condition and clinical manifestations and signs of the patient, supported by the CT image of the neck, emergency surgery was carried out to incise and discharge the abscess (Figure 1C). All the patients were treated initially with amoxicillin/clavulanic acid and metronidazole, with treatment adjusted according to subsequent culture and sensitivity reports. Vascular forceps were used for blunt separation and wide opening of the dangerous abscess cavity that was then explored using the fingers to ensure the pus had been drained and necrotic tissues removed as much as possible (Figure 1D). The abscess cavity was washed repeatedly with hydrogen peroxide, diluted complex iodine, and normal saline.

Patients in group A group were treated by oxygen therapy of the pus cavity. On the first day after the operation, an oxygen tube was placed in the pus cavity with several side holes opened in the front of the oxygen tube (Figure 2A). The oxygen flow rate was maintained at $1-4$ $\mathrm{L} / \mathrm{min}$ for $8-12 \mathrm{~h} /$ day. When there was no obvious purulent secretion, the incision was sutured and negative pressure drainage started (Figure 2B). The color of the drainage fluid was observed every day. When the color of the fluid was clear and its volume $<10 \mathrm{~mL}$, a further CT of the neck was performed to confirm that the abscess

Table I Distribution of the Types of Deep Neck Infections

\begin{tabular}{|l|c|c|c|}
\hline Diagnosis & Total $\mathbf{n}$ (\%) (N= 36) & Group A n (\%) (N= 13) & Group B n (\%) (N= 23) \\
\hline Parapharyngeal space infection & $20(55.6)$ & $8(61.5)$ & $12(52.3)$ \\
Submandibular space infection & $7(19.4)$ & $3(23.1)$ & $4(17.4)$ \\
Multi-space infection & $6(16.7)$ & $1(7.7)$ & $5(21.7)$ \\
Necrotizing myofasciitis & $2(5.6)$ & $1(7.7)$ & $1(4.3)$ \\
Retropharyngeal space infection & I (2.8) & 0 & $1(4.3)$ \\
\hline
\end{tabular}




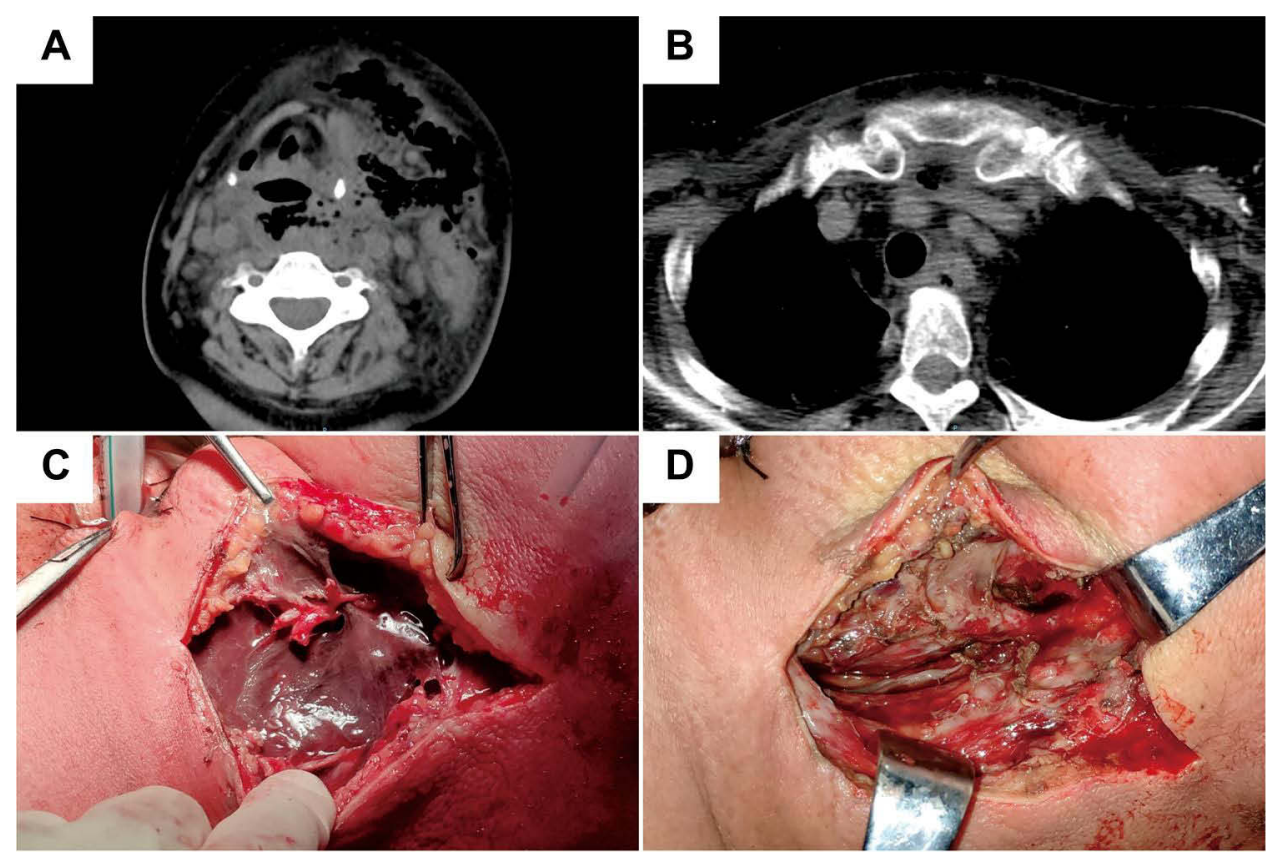

Figure I CT images of the neck showing low density shadowing and a gas shadow in the cervical subcutaneous, parapharyngeal, retropharyngeal, prevertebral, carotid, and upper mediastinum spaces (A and B). Necrotic fascia of the sternocleidomastoid muscle (C). Removal of necrotic tissue followed by flushing of the pus cavity (D).
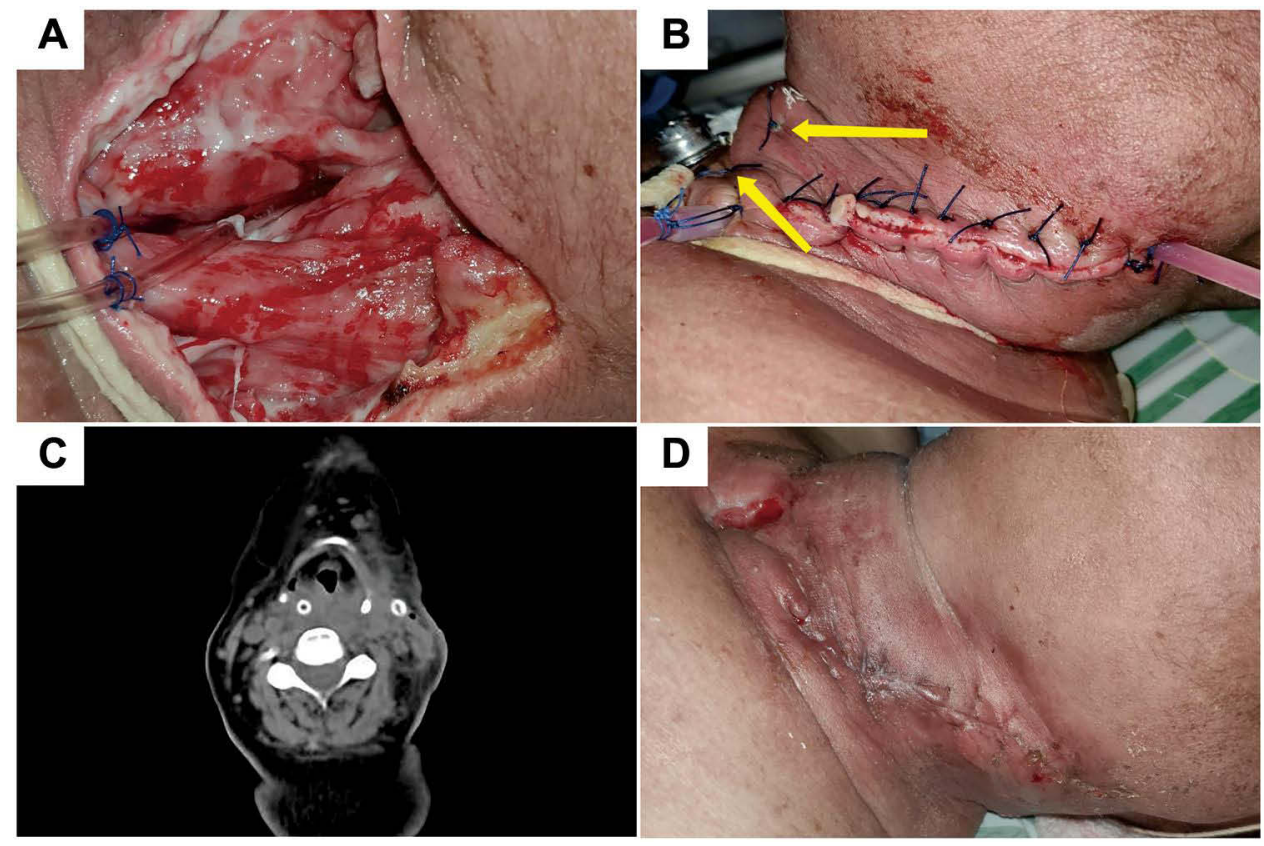

Figure 2 An oxygen tube placed in the pus cavity (A). The pus cavity was sutured and a negative pressure drainage placed in position (B). A re-examination CT image of the neck showing that the pus cavity had disappeared and the swelling of soft tissue had subsided (C). Following removal of the drainage tube the incision healed well (D).

cavity had disappeared. The negative pressure drainage tube was then removed (Figure 2C). The patient was cured and discharged (Figure 2D).

In group $\mathrm{B}$, the dressing was changed daily to wash the pus cavity and place the drainage gauze in position. The dressing was changed twice a day if too much pus was in the cavity, with the frequency of dressing changes reduced according to the clinical situation. The incision was sutured when the wound had developed fresh granulation tissue with no obvious purulent exudate. If a refractory skin defect was present, a flap transfer was sometimes required to repair the defect. 
Table 2 Comparison of Treatment Effects Between the Two Treatment Groups ( $\overline{\mathrm{X}} \pm \mathrm{s})$

\begin{tabular}{|l|c|c|c|}
\hline Group & $\mathbf{n}$ & Average Hospitalization Days (d) & Average Days of Postoperative Dressing Change (d) \\
\hline Group A & 13 & $11.08 \pm 2.11$ & $3.69 \pm 0.21$ \\
Group B & 23 & $26.74 \pm 3.39$ & $25.91 \pm 3.43$ \\
P & & 0.0025 & $<0.0001$ \\
\hline
\end{tabular}

All patients had a pus culture and antimicrobial susceptibility test carried out during the course of treatment.

\section{Results}

The statistical analyses were performed using GraphPad Prism 6 (GraphPad Prism 6 Software, San Diego, CA, USA). A comparison of the average hospitalization days and average days of postoperative dressing changes between the two groups was performed using Student's $t$-test. The two indices were lower in group $\mathrm{A}$ than in group B. P values $<0.05$ were considered statistically significant (Table 2). All 36 patients were cured following treatment.

In group B, 7 of the 23 patients (30.4\%) had early skin defects, 5 of which healed spontaneously, while 2 cases were healed by a transfer skin flap. No skin defect occurred in group A.

All 36 patients had a pus culture, with 3 cases of Staphylococcus aureus, 1 case of Streptococcus pyogenes, 1 case of Klebsiella pneumoniae, and 32 cases of a negative culture result.

\section{Discussion}

DNSI are defined by the presence of inflammation with or without pus in the deep spaces and fasciae of the head and neck. ${ }^{7}$ A DNSI is often secondary to an infection of the tonsils, teeth, and upper respiratory tract or may develop following deep neck surgery. ${ }^{8}$ If the infection cannot be controlled in time it can spread to other deep neck spaces, even up to the skull base or down to the mediastinum, or alternatively cause other abscesses and even systemic symptoms. A DNSI may also lead to upper respiratory tract obstruction, eventually causing serious consequences or even death. ${ }^{9}$ Therefore, early incision and drainage should be performed if the diameter of an abscess is confirmed by imaging to be $>2.5 \mathrm{~cm} .{ }^{10}$ Cramer et $\mathrm{al}^{4}$ reported that delay in surgical drainage of deep neck abscess was associated with increased abscess-specific morbidity and mortality in their study.
The most common site of infection in our study was the parapharyngeal space $(55.6 \%)$, a finding consistent with the results of Huang et al. ${ }^{11}$ This occurs because the parapharyngeal space is often involved with the peritonsillar, submandibular, masticatory, and parotid space. ${ }^{2}$ A DNSI can be a single or mixed infection of a variety of microorganisms with Streptococcus sp., digestive streptococcus, Staphylococcus aureus, and anaerobes being the most common bacteria cultured from DNSI. ${ }^{12}$ Bacterial cultures of pus are often negative as a result of early application of antibiotics or improper sampling of materials and other factors and are therefore used only as a reference for antibiotic therapy. A study by Walia et $\mathrm{al}^{6}$ reported that 10 anaerobic microorganisms $(25 \%)$ were isolated in 40 cultures. However, because we did not perform anaerobic cultures we have no data on the possible existence of anaerobes in the pus samples. Heim et al showed that about $42 \%$ of patients had at least one bacterial strain that showed antibiotic resistance, ${ }^{13}$ and proposed that immediate surgical treatment involving incision and drainage formed the basis for treatment of an abscess, with antibiotics acting as adjunct therapy.

A large amount of pus may be produced every day after the abscess cavity is opened in DNSI patients. In patients with necrotizing myofasciitis, the initial opening of the infected cavity results in very little pus, although exposure of the infected cavity to air can subsequently produce a large amount of pus. Cao et al reported that the median volume of drainage fluid within 3 days after surgery was $420 \mathrm{~mL}$, ranging between 280 and $760 \mathrm{~mL}{ }^{14}$

In view of this situation, we used the pyogenic cavity aerobic therapy which involved passing an oxygen tube into the operation cavity and adjusting the oxygen flow to 1-3 L/min. After the first use of this therapy, the exudation of pus was reduced to about $10-15 \mathrm{~mL}$ the following day, and according to the amount of exudation in the operation cavity the oxygen flow was then gradually reduced until all the pus had been removed. During this period, we observed that if the oxygen flow rate was adjusted to more than $4 \mathrm{~L} / \mathrm{min}$, the operation cavity became too dry 
causing the gauze to adhere to the deep neck tissue making it difficult to separate. This situation was not conducive to the growth of granulation tissue and wound healing. Secondly, during the period of aerobic treatment, the neck bandage should be loose in order to facilitate the outflow of oxygen and prevent the infection from spreading to the surrounding spaces due to excessive air pressure. The oxygen flow tube should also not be plugged into the dead cavity in order to avoid serious consequences. When the drainage fluid is clear or its volume reduced to $<10 \mathrm{~mL}$, the fluid should be removed using negative pressure drainage, followed by suturing of the operation cavity. If the pus cavity is connected with the gas incision stoma, the cavity needs to be closed by a suture (Figure 2B, shown by the yellow arrow) to avoid sputum draining into the infection cavity during negative pressure drainage.

Previous treatment methods in patients with a DNSI mainly involved incision and drainage of pus and changes of dressings up to two times a day until the pus has disappeared or granulation tissue has filled the operation cavity. The incision is then sutured or the dressing changed until the incision has healed which often takes about half a month to a few months. The majority of first-line clinical doctors still use this traditional flushing and dressing change treatment. By using the pyogenic cavity aerobic therapy with negative pressure drainage approach, the volume and characteristics of the drainage fluid only need to be observed daily, considerably reducing the psychological and physical trauma of patients and workload of clinicians.

It is important that patients pay close attention to their general health status, especially blood glucose and blood pressure levels. Without good blood glucose control, ketoacidosis, the hypertonic syndrome, lactic acidosis or hypoglycemia may occur, conditions which are not conducive to follow-up treatment. In the current study, 21 cases (58.3\%) had type 2 diabetes with the majority having large fluctuations in blood glucose levels with poor control. After admission, it is necessary to actively contact an endocrinologist for consultation and adjust the dosages of hypoglycemic drugs especially in patients with oral feeding difficulties who require nasal feeding nutrition. We chose insulin to control blood glucose levels as much as possible. Based on the results of blood glucose monitoring the type and dosage of insulin should be adjusted in a timely manner.
Nutritional support is very important in patients with a DNSI. Because the lesions are located in the neck and oropharynx, many patients have oral feeding difficulties. In this regard, an indwelling gastric tube for nasal feeding nutrition can be used to provide enteral nutrition fluids of between $500-1500 \mathrm{~mL}$ every day. Family members of patients should also be provided with guidance on nasal feeding. During this period, routine blood and biochemical indexes should be reexamined daily or every other day to monitor the infection index, electrolyte status, and the nutritional status of patients. Patients with poor general health, especially those with poor cardiopulmonary function, may need to be transferred to the intensive care department for further treatment.

It has been reported that a drainage tube placed in the pus cavity can be used to treat infections of the deep neck space due to their good efficacy and cosmetic effects. ${ }^{15,16}$ This technique is therefore an appropriate treatment strategy for a small single abscess. Reports have shown that vacuum sealing drainage (VSD) or a method similar to VSD can markedly improve the therapeutic effect of deep neck multiple space infections. ${ }^{9,14}$ Cortese et $\mathrm{al}^{5}$ reported that the use of a suctioning resection technique associated with hyperbaric oxygen therapy achieved good results in the treatment of odontogenic necrotizing fasciitis. They considered that sucking and removing dead tissue controlled the spread of inflammation into deeper areas. ${ }^{5}$ Crew et $\mathrm{al}^{17}$ reported that negative pressure wound therapy combined with irrigation of a pure hypochlorous acid solution $0.01 \%$ achieved good results in the treatment of acute necrotizing fasciitis, as this combination therapy killed bacteria quickly and simultaneously inactivated toxins and superantigens. ${ }^{17} \mathrm{We}$ consider that when an abscess in the deep neck spaces has formed in a large area, incision and drainage should be carried out as early as possible, especially for a multiple lacunar abscess. During the operation, all the septa should be separated as far as possible without leaving a dead space and the necrotic tissue removed completely whilst preserving important nerves and blood vessels. Pyogenic cavity aerobic therapy with negative pressure drainage is more straightforward and effective and easy to operate compared to the approaches reported in other studies.

CT scanning is the most widely used procedure for diagnosing a DSNI because it is cheap, easily available, and can locate head and neck abscesses and other structural abnormalities. CT combines fast image acquisition 
and precise anatomical information without field-of-view limitations. Nurminen et $\mathrm{al}^{18}$ reported that magnetic resonance imaging (MRI) had very high diagnostic accuracy and predictive value for both infections and abscesses, whereas MRI was more complex and time-consuming to read than $\mathrm{CT}$. For these reasons, $\mathrm{CT}$ is the first imaging examination of choice in emergency situations, especially in cases of shortness of breath. If an abscess has not yet formed or the scope is small, the patients can be followedup by CT examination or color Doppler ultrasound examination according to the general clinical situation.

Once a diagnosis of DNSI is made, it is necessary to first closely monitor the patient's respiratory status and if dyspnea occurs evaluate whether a tracheotomy is necessary. Local anesthesia is recommended with the help of an anesthesiologist because the patient may have airway distortion, tissue stiffness, and limited mouth opening. ${ }^{3}$ Laryngoscope tracheal intubation should not be used because intubation may further damage the airway. ${ }^{12}$ However, if airway conditions and anesthesia conditions permit, intubation can also be attempted. If intubation fails, a tracheotomy should be performed urgently to ensure the safety of patients.

Compared with the traditional treatment of daily irrigation and dressing changes, the abscess cavity aerobic treatment provides high concentrations of oxygen to the local infected necrotic tissue, which not only promotes healing of the tissue, but also effectively inhibits the growth of anaerobic bacteria. The continuous circulation of the abscess cavity also effectively evaporates the fluid secreted by the abscess cavity and keeps the wound clean. When the pus decreases or disappears, the combination of suture incision and continuous negative pressure drainage can effectively avoid the occurrence of skin defects and achieve better cosmetic results.

\section{Conclusions}

The pyogenic cavity aerobic therapy and incision and drainage of pus described in this paper involves a simple operation process and has proved to be a good treatment method for patients with DNSI. Combined with negative pressure drainage, this therapy shortens the average hospitalization days and reduces the physical and psychological pain of patients. The method not only reduces the workload of clinical doctors but also avoids the occurrence of skin defects, reduces the risk of rupturing large blood vessels and damaging important nerve structures during changes of dressings. The materials used in the treatment process are also common clinical materials. We therefore consider that this method is worthy of clinical promotion in some cases.

\section{Acknowledgments}

The authors would like to express their gratitude to EditSprings for the expert linguistic services provided.

\section{Author Contributions}

All authors made a significant contribution to the work reported, whether that is in the conception, study design, execution, acquisition of data, analysis and interpretation, or in all these areas; took part in drafting, revising or critically reviewing the article; gave final approval of the version to be published; have agreed on the journal to which the article has been submitted; and agree to be accountable for all aspects of the work.

\section{Disclosure}

The authors report no conflicts of interest in this work.

\section{References}

1. Opitz D, Camerer C, Camerer DM, et al. Incidence and management of severe odontogenic infections - a retrospective analysis from 2004 to 2011. J Craniomaxillofac Surg. 2015;43(2):285-289. doi:10.1016/ j.jcms.2014.12.002

2. Wu JH, Li X, Chen GP, Fu MY, Ye F. Changing trends of deep neck infections in southern China: a review of 127 cases. Sci Prog. 2021;104(2):368504211028367. doi:10.1177/00368504211028367

3. Osborn TM, Assael LA, Bell RB. Deep space neck infection: principles of surgical management. Oral Maxillofac Surg Clin North Am. 2008;20(3):353-365. doi:10.1016/j.coms.2008.04.002

4. Cramer JD, Purkey MR, Smith SS, Schroeder JW Jr. The impact of delayed surgical drainage of deep neck abscesses in adult and pediatric populations. Laryngoscope. 2016;126(8):1753-1760. doi:10.1002/lary.25835

5. Cortese A, Pantaleo G, Borri A, Amato M, Claudio PP. Necrotizing odontogenic fasciitis of head and neck extending to anterior mediastinum in elderly patients: innovative treatment with a review of the literature. Aging Clin Exp Res. 2017;29(Suppl 1):159-165. doi:10.1007/s40520-016-0650-2

6. Walia IS, Borle RM, Mehendiratta D, Yadav AO. Microbiology and antibiotic sensitivity of head and neck space infections of odontogenic origin. J Maxillofac Oral Surg. 2014;13(1):16-21. doi:10.1007/ s12663-012-0455-6

7. Beka D, Lachanas VA, Doumas S, et al. Microorganisms involved in deep neck infection (DNIs) in Greece: detection, identification and susceptibility to antimicrobials. BMC Infect Dis. 2019;19(1):850. doi:10.1186/s12879-019-4476-3

8. Parhiscar A, Har-El G. Deep neck abscess: a retrospective review of 210 cases. Ann Otol Rhinol Laryngol. 2001;110(11):1051-1054. doi:10.1177/000348940111001111

9. Gu X, Chen W, Yuan K, Tan J, Sun S. The efficacy of artificial dermis combined with continuous vacuum sealing drainage in deep neck multiple spaces infection treatment. Medicine. 2021;100(5): e24367. doi:10.1097/MD.0000000000024367

10. Wilkie MD, De S, Krishnan M. Defining the role of surgical drainage in paediatric deep neck space infections. Clin Otolaryngol. 2019;44 (3):366-371. doi:10.1111/coa.13315 
11. Huang TT, Liu TC, Chen PR, Tseng FY, Yeh TH, Chen YS. Deep neck infection: analysis of 185 cases. Head Neck. 2004;26 (10):854-860. doi:10.1002/hed.20014

12. Gujrathi AB, Ambulgekar V, Kathait P. Deep neck space infection a retrospective study of 270 cases at tertiary care center. World $J$ Otorhinolaryngol Head Neck Surg. 2016;2(4):208-213. doi:10.1016/j.wjorl.2016.11.003

13. Heim N, Jürgensen B, Kramer FJ, Wiedemeyer V. Mapping the microbiological diversity of odontogenic abscess: are we using the right drugs? Clin Oral Investig. 2021;25(1):187-193. doi:10.1007/ s00784-020-03350-0

14. Cao J, Liu Z, Ma D, Shen S, Wang X. Modified usage of negative pressure wound therapy for the management of severe deep fascial space infections in the head and neck. Infect Drug Resist. 2020;13:781-788. doi:10.2147/IDR.S243794
15. Tanaka K, Tsunoda A, Tou M, et al. Minimally invasive and inexpensive percutaneous abscess drainage using an indwelling needle cannula. Am J Otolaryngol. 2020;41(6):102664. doi:10.1016/j. amjoto.2020.102664

16. Hawkins A, Ponnusamy S, Markiewicz MR, Callahan N. Use of a Foley catheter in the drainage of deep space neck infections. $\mathrm{Br}$ $J$ Oral Maxillofac Surg. 2019;57(9):942-943. doi:10.1016/j. bjoms.2019.07.016

17. Crew JR, Varilla R, Allandale Rocas Iii T, Abdul RS, Debabov D. Treatment of acute necrotizing fasciitis using negative pressure wound therapy and adjunctive neutrophase irrigation under the foam. Wounds. 2013;25(10):272-277.

18. Nurminen J, Velhonoja J, Heikkinen J, et al. Emergency neck MRI: feasibility and diagnostic accuracy in cases of neck infection. Acta Radiol. 2021;62(6):735-742. doi:10.1177/0284185120940242

\section{Publish your work in this journal}

Infection and Drug Resistance is an international, peer-reviewed openaccess journal that focuses on the optimal treatment of infection (bacterial, fungal and viral) and the development and institution of preventive strategies to minimize the development and spread of resistance. The journal is specifically concerned with the epidemiology of antibiotic resistance and the mechanisms of resistance development and diffusion in both hospitals and the community. The manuscript management system is completely online and includes a very quick and fair peerreview system, which is all easy to use. Visit http://www.dovepress.com/ testimonials.php to read real quotes from published authors. 